\title{
Torpedo Maculopathy Associated with RP1L1 Mutation and Rod-Cone Dystrophy
}

\author{
Manoj Soman ${ }^{1,2}$, Jay U Sheth ${ }^{1,2 *}$, Asmita Indurkar ${ }^{1,2}$ and Unnikrishnan \\ Nair $^{1,2}$ \\ ${ }^{1}$ Vitreoretinal Services, Chaithanya Eye Hospital and Research Institute, India \\ ${ }^{2}$ Chaithanya Innovation in Technology and Eyecare (Research), India \\ *Corresponding Author: Jay U Sheth, Clinical Research Lead, Vitreoretinal Services, \\ Chaitanya Eye Hospital and Research Institute, Trivandrum, India.
}

Received: March 12, 2021

Published: April 23, 2021

(C) All rights are reserved by Jay U Sheth., et al.

\begin{abstract}
Genetic analysis of patients with Torpedo maculopathy is scarce in literature with only two previously documented cases with genetic analysis. We report a case of Torpedo maculopathy with Rod-Cone dystrophy associated with an RP1L1 mutation. This case highlights the need for genetic testing in this rare retinal pathology as this may give a clue to the presence of other ocular associations and systemic abnormalities.
\end{abstract}

Keywords: Torpedo Maculopathy; RP1L1 Mutation; Rod-Cone Dystrophy; Genetic Testing; Electroretinogram

\section{Abbreviations}

RPE: Retinal Pigment Epithelium; RP1L1: Retinitis Pigmentosa1-Like-1; SD-OCT: Spectral-Domain Optical Coherence Tomography; VEP: Visual Evoked Potential; MRI: Magnetic Resonance Imaging; VUS: Variant of Uncertain Significance; ERG: Electroretinogram; LVA: Low Vision Aid; CNVM: Choroidal Neovascular Membrane; OS: Outer Segment; OMD: Occult Macular Dystrophy

\section{Introduction}

Torpedo maculopathy is a rare, incidental retinal finding believed to a presumably congenital lesion involving the retinal pigment epithelium (RPE) [1]. Retinal laser ellipsometry analysis has revealed a kite-shaped region at the junction of superior arcuate, inferior arcuate, and papillomacular bundle to be the location of this finding, a region of embryologically sparse nerve fiber layers [2]. Genetic analysis of these patients however is scarce in the literature. The only 2 reported cases with genetic analysis include one with NEXMIF mutation and the other with TSC2 gene mutation $[3,4]$. We report a case of Torpedo maculopathy associated with an RP1L1 (Retinitis Pigmentosa-1-Like-1) mutation not hitherto reported in the literature.

\section{Case Report}

A 2-year-old male child presented to our clinic with bilateral abnormal eye movements since birth. He was born of a full-term normal delivery with a birth weight of $3.2 \mathrm{~kg}$ in a consanguineous family. He had a normal perinatal course and was immunized for age. From a developmental perspective, he was diagnosed to have a global delay by the pediatrician. On examination, he was fixing and following the light and was noted to have large amplitude pendular nystagmus. Retinoscopy did not reveal any gross refractive error. Anterior segment examination was unremarkable with normal pupils in both eyes. Fundus examination revealed a large pale disc with myelinated nerve fibers inferior to the disc in both eyes. A torpedo-shaped hypopigmented atrophic patch with well-defined margins was noted inferotemporal to right eye fovea (Figure 1). Spectral-domain optical coherence tomography (SDOCT) of the right eye (Figure 2) revealed type 1 pattern [5] with inner segment-outer segment irregularity, attenuation of the ellip- 
soid layer and interdigitation zone, RPE thinning and RPE choroidal excavation without any outer retinal cavitation. A flash visual evoked potential (VEP) was performed in view of disc pallor and for assessment of his visual acuity, which showed irregular waveforms (Figure 3a). Pediatric neurology evaluation was done, and magnetic resonance imaging (MRI) brain was normal. As a part of his systemic evaluation, chromosomal sequencing was done which revealed a heterozygous 'variant of uncertain significance' (VUS) variant in the RP1L1 gene (Figure 4). A flash electroretinogram (ERG) revealed suppression of scotopic and photopic waveforms in both eyes suggestive of Rod-Cone dystrophy (Figure 3b). The patient has been advised low vision aid (LVA) along with adaptive training and followed up at 6 months interval. Genetic counseling was undertaken for the family.

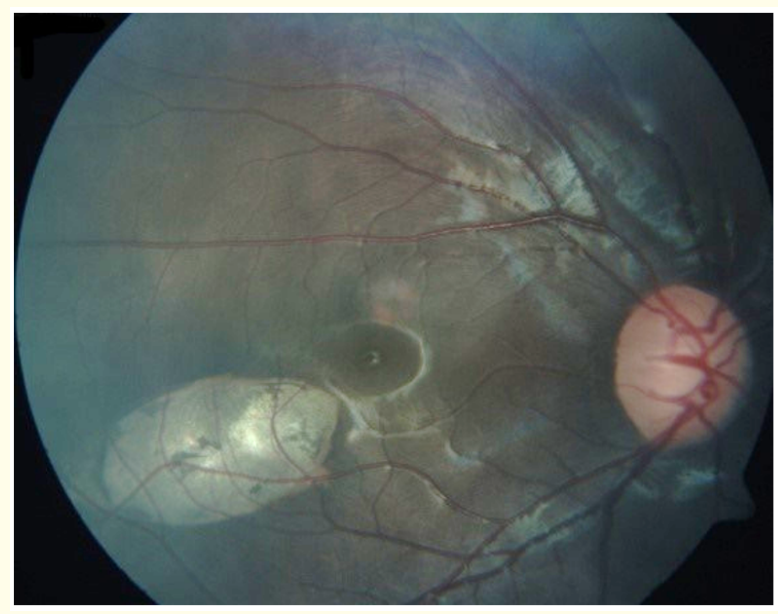

Figure 1: Fundus picture of the right eye showing torpedo-shaped hypopigmented atrophic patch with well-defined margins inferotemporal to the fovea.

\section{Discussion}

We report a case of Torpedo maculopathy associated with an RP1L1 mutation and Rod-Cone dystrophy. The patient had a type 1 pattern of Torpedo maculopathy with an absence of any systemic associations.

Multiple hypotheses have been proposed for the etiology of Torpedo maculopathy, including a persistent developmental defect in the RPE in the location of temporal fetal bulge, abnormal ciliary or choroidal vascular development, or incomplete arcuate bundle differentiation along the horizontal raphe $[1,6,7]$ Although the exact

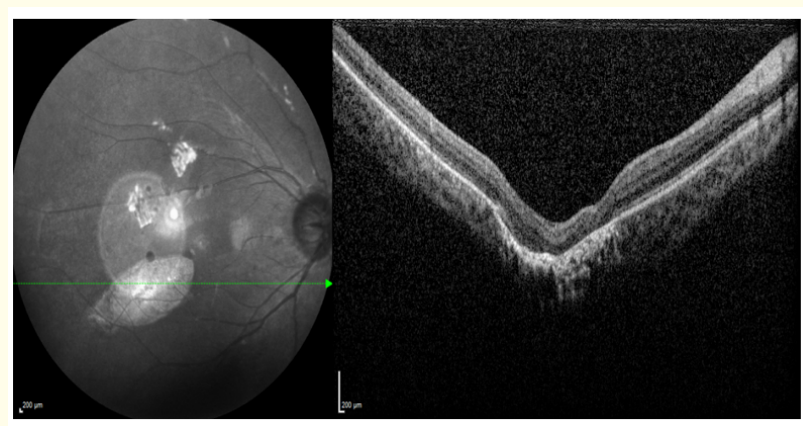

Figure 2: Spectral-domain optical coherence tomography (SD-OCT) scan through Torpedo maculopathy lesion showing normal inner retinal layers, outer segment abnormality with loss of ellipsoid layer, interdigitation zone, RPE thinning, and

RPE- choroidal excavation with no outer retinal cavitation

(Type 1 Pattern).

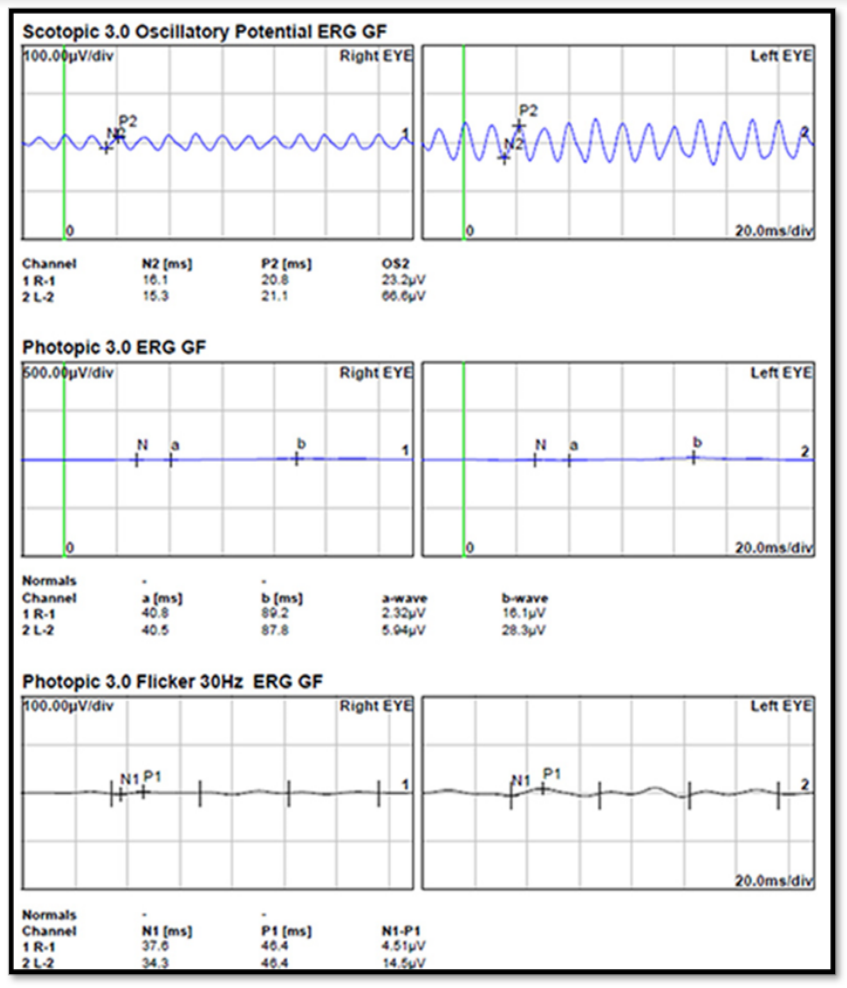

Figure 3a: Electrophysiological Investigations - a: Flash VEP showing abnormal irregular waveforms. 


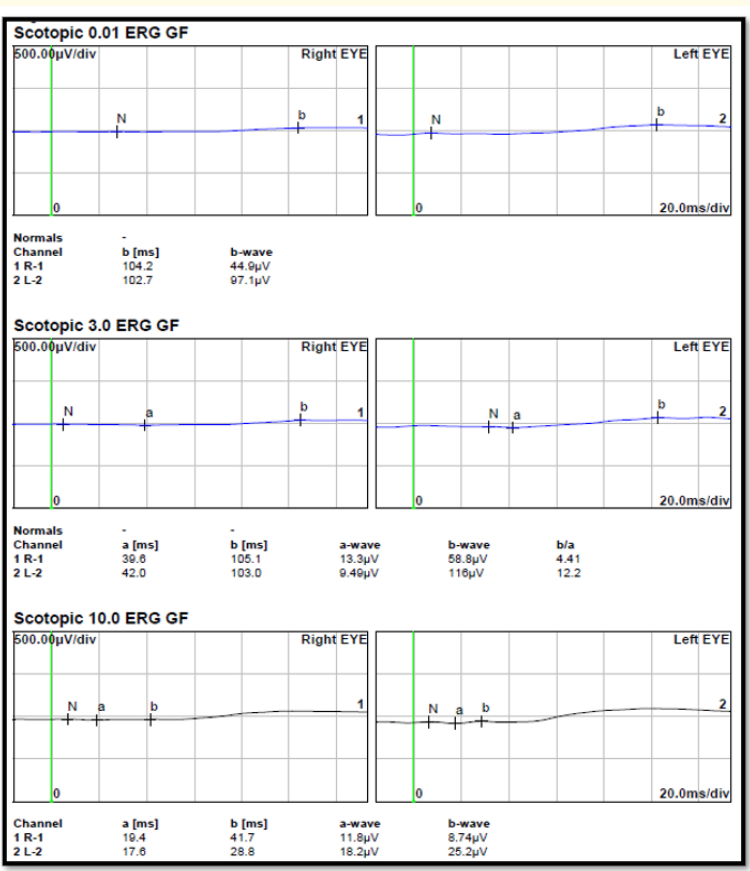

Figure 3b: Flash ERG revealed suppression of both scotopic and photopic waveforms suggestive of rod-cone dysfunction.

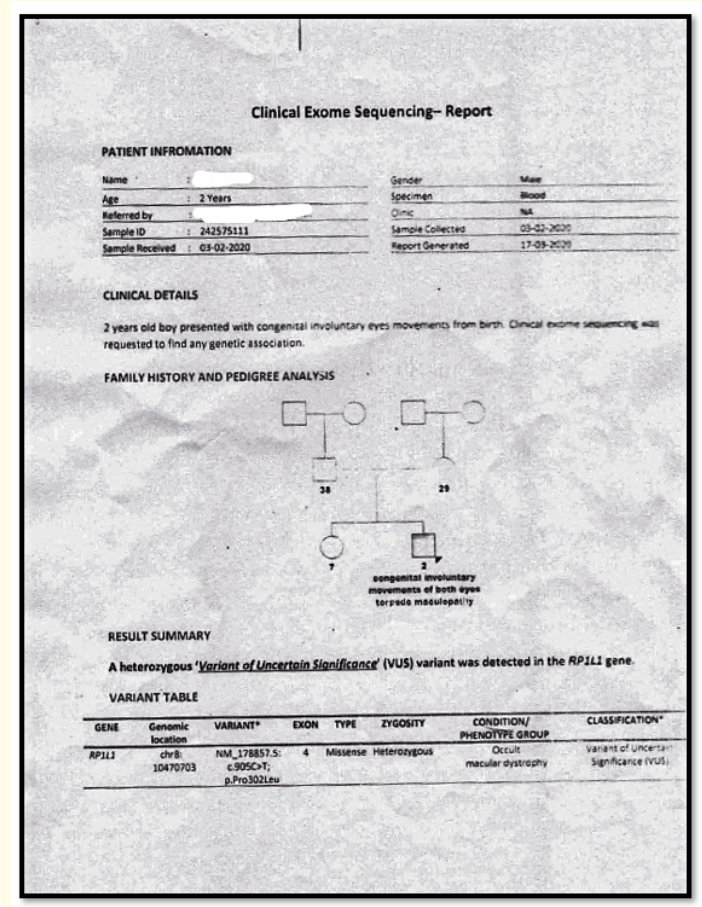

Figure 4: Chromosomal sequencing revealing a heterozygous 'variant of uncertain significance' (VUS) variant in the RP1L1 gene. pathogenesis remains elusive, it is universally accepted that Torpedo maculopathy is a benign congenital lesion with a non-progressive course. Very few case reports have shown the occurrence of a choroidal neovascular membrane (CNVM) in Torpedo lesions $[8,9]$. Hence, regular follow-up of these patients is vital.

Our patient had an RP1L1 mutation on genetic testing. In the literature, genetic analysis studies have revealed an association of Torpedo maculopathy with NEXMIF mutation and others with TSC2 gene mutation $[3,4]$. These cases of Torpedo maculopathy with reported mutations had other systemic manifestations consistent with the mutations including autistic features and mental retardation in the former and tuberous sclerosis with a retinal astrocytoma in the latter report [3,4]. In our patient, there were no other systemic associations other than a global developmental delay. Also, most case reports and reviews on Torpedo maculopathy have not highlighted the importance of genetic testing or observation of associated ocular findings or systemic associations $[10,11]$.

The RP1L1 gene is a component of the photoreceptor cilium and encodes a component of the photoreceptor axoneme [12]. This axoneme is vital for maintaining the biochemical homeostasis of the photoreceptor outer segment (OS) by allowing transportation between the OS and inner segment and for the construction of OS discs [12]. Any pathogenic variation in this RP1L1 gene can thus lead to photoreceptor degenerative disorders. The known association of RP1L1 mutations include occult macular dystrophy (OMD), Autosomal recessive Retinitis pigmentosa, Adult pseudovitelliform dystrophy, severe macular degeneration, and rod-cone dystrophy $[12,13]$. As observed in these case reviews with RP1L1 mutations $[12,13]$, our case also had abnormal ERG even though clinically documented pigmentary maculopathy or retinopathy was absent. Additionally, our case also had the presence of Torpedo maculopathy in association with RP1L1 mutation, which has not been reported earlier. The association of Torpedo maculopathy with this mutation may likely be a chance occurrence. However, since many other retinal anomalies have been reported with this mutation [12,13], a possibility of concurrent embryological malformation giving rise to Torpedo maculopathy cannot be completely ruled out.

With this report and review of the previous reports with genetic analysis, we would like to emphasize that it is necessary to do a genetic analysis in all patients who are diagnosed to have Torpedo maculopathy. Identification of the genetic mutation could help us understand the other systemic or ocular pathologies that may be associated and help us understand the related embryological events. We advised the ERG in our case solely based on the positive 
genetic analysis, which yielded significant rod-cone dysfunction. We recommend a pooled genetic analysis of the reported cases of Torpedo maculopathy to help us better understand the genetic predisposition of these rare eyes.

\section{Conclusion}

In conclusion, this case highlights three important observations. Firstly, multiple genetic mutations may be associated with torpedo maculopathy. Secondly, subjects with torpedo maculopathy with genetic mutations must be tested electrophysiologically as there could be concurrent occult retinal dystrophies, and lastly, these subjects should also be appropriately investigated systemically as they may be associated with neurologic abnormalities. All these will help in identifying the disease spectrum and recommend timely vision therapy before dense amblyopia may set in. With these measures, we can improve the quality of life of these visually impaired children.

\section{Conflict of Interest}

No financial interest or any conflict of interest exists.

\section{Bibliography}

1. Golchet PR., et al. "Torpedo maculopathy". British Journal of Ophthalmology 94.3 (2010): 302-306.

2. Dreher AW., et al. "Spatially resolved birefringence of the retinal nerve fiber layer assessed with a retinal laser ellipsometer". Applied Optics 31.19 (1992): 3730-3735.

3. Alarcon-Martinez T., et al. "Torpedo Maculopathy Associated with NEXMIF Mutation". Molecular Syndromology 10.4 (2019): 229-233.

4. Hansen MS., et al. "Optical coherence tomography of torpedo maculopathy in a patient with tuberous sclerosis". Acta Ophthalmologica 94.7 (2016): 736-737.

5. Wong EN., et al. "Novel optical coherence tomography classification of torpedo maculopathy". Clinical and Experimental Ophthalmology 43.4 (2015): 342-348.

6. Shields CL., et al. "Torpedo maculopathy at the site of the fetal "bulge". Archives of Ophthalmology 128.4 (2010): 499-501.

7. Pian D., et al. "Paramacular coloboma". Optometry and Vision Science 80.8 (2003): 556-563.
8. Parodi MB., et al. "Choroidal Neovascularization in Torpedo Maculopathy Assessed on Optical Coherence Tomography Angiography". Ophthalmic Surgery, Lasers and Imaging Retina 49.11 (2018): e210-e213.

9. Shirley K., et al. "Torpedo maculopathy: disease spectrum and associated choroidal neovascularisation in a paediatric population". Eye 32.8 (2018): 1315-1320.

10. Williams PJ., et al. "Distribution patterns of torpedo maculopathy: Further evidence of a congenital retinal nerve fiber layerdriven etiology". Saudi Journal of Ophthalmology 33.3 (2019): 260-267.

11. Soman M., et al. "Indocyanine green angiography and multimodal imaging in a case of torpedo maculopathy". Indian Journal of Ophthalmology 68.7 (2020): 1448-1449.

12. Noel NCL and MacDonald IM. "RP1L1 and inherited photoreceptor disease: A review". Survey of Ophthalmology 65.6 (2020): 725-739.

13. Akahori M., et al. "Dominant mutations in RP1L1 are responsible for occult macular dystrophy". American Journal of Human Genetics 87.3 (2010): 424-429.

\section{Assets from publication with us}

- Prompt Acknowledgement after receiving the article

- Thorough Double blinded peer review

- Rapid Publication

- Issue of Publication Certificate

- High visibility of your Published work

Website: www.actascientific.com/

Submit Article: www.actascientific.com/submission.php

Email us: editor@actascientific.com

Contact us: +919182824667 